\title{
Planification de trajectoire par champs de potentiel fractionnaires appliquée au véhicule autonome
}

\section{Path planning with fractional potential fields for autonomous vehicles}

\author{
Julien Moreau ${ }^{1,2,3}$, Pierre Melchior ${ }^{1}$, Stéphane Victor ${ }^{1}$, Mathieu Moze ${ }^{2}$, François Aioun², \\ Franck Guillemard ${ }^{2}$
}

${ }^{1}$ IMS - UMR 5218 CNRS, Université de Bordeaux/Bordeaux INP

351 cours de la Libération, 33405 Talence cedex - France

Email : prenom.nom@ims-bordeaux.fr

${ }^{2}$ PSA Groupe

Chemin de Gisy, 78140 Vélizy-Villacoublay cedex - France

Email : prenom.nom@mpsa.com

${ }^{3}$ OpenLab PSA Groupe-IMS Bordeaux

\begin{abstract}
RÉSUMÉ. La planification de trajectoire est une partie essentielle pour le contrôle des robots mobiles. Ceci est plus vrai que jamais dans le contexte automobile et tout particulièrement pour le véhicule autonome. Dans ce papier, la méthode des champs de potentiel est proposée afin de respecter ces contraintes. De plus, les véhicules autonomes sont considérés équipés de tous les capteurs nécessaires pour la détection d'obstacles. Ainsi, les champs de potentiel attractif de Ge\&Cui et les champs de potentiel attractif fractionnaire ont été adaptés au contexte du véhicule autonome permettant ainsi d'assurer une meilleure robustesse du degré de stabilité tout en contrôlant l'accélération du véhicule.

ABSTRACT. Path planning is an essential stage for mobile robot control. It is more newsworthy than ever in the automotive context and especially for autonomous vehicle. Also, path planning methods need to be adaptive regarding life situations, traffic and obstacle crossing. In this paper, potential field methods are proposed to cope with these constraints and autonomous vehicles are considered equipped with all necessary sensors for obstacle detection. In this way, Ge\&Cui's attractive potential field and fractional attractive potential field have been adapted to the context of autonomous vehicles. In this way, this latter method ensures better stability degree robustness with controlled vehicle acceleration.

MOTS-CLÉS. Planification de trajectoire, automobile, véhicule autonome, champs de potentiel, champs de potentiel fractionnaire.

KEYWORDS. Path planning, automotive, autonomous vehicle, potential field, fractional potential field.
\end{abstract}

\section{Introduction}

La conduite autonome va devenir le coeur des transports publics dans le futur. Le véhicule autonome a le potentiel d'améliorer la sécurité des passagers, de réduire le temps de voyage et d'améliorer le confort des passagers. Par conséquent, les fabricants d'automobiles investissent dans la conception et l'équipement de leur véhicule avec des systèmes avancés d'aide à la conduite (ADAS). Dans les zones urbaines, les intersections sont considérées comme des goulots d'étranglement. En 2004, plus de $43 \%$ des accidents de la route reportés en Europe ont lieu en intersection et sont responsables de 46,821 morts ainsi qu'approximativement 293,000 blessés en un an [Lefèvre(2013)]. De plus, les retards dus au trafic en intersection conduisent à une énorme perte de ressources. Par conséquent, l'utilisation des véhicules autonomes est une solution à ces problèmes.

La zone urbaine représente le cas critique pour le véhicule autonome. En effet, ce genre d'environnement est très dynamique : le véhicule autonome doit être adaptatif vis-à-vis des situations de vie, du trafic et des différents obstacles. Dans ce papier, les véhicules autonomes sont considérés équipés de tous les capteurs nécessaires pour la détection d'obstacles.

Ces dernières années, la planification de trajectoire pour les véhicules autonomes a été largement étu- 


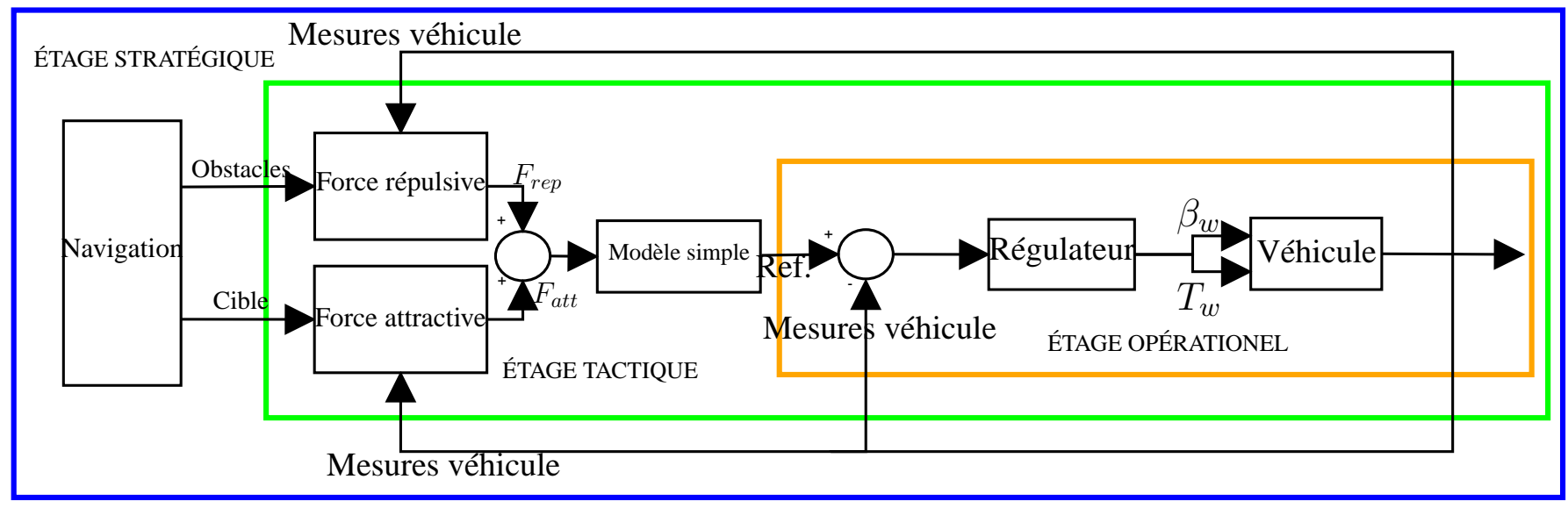

Figure 1. Schéma de régulation à trois niveaux : stratégique (bleu), tactique (vert) et opérationel (orange))

diée dans la littérature. Dans [Makarem(2011)], une fonction de navigation pour les véhicules autonomes a été développée mais aucun piéton n'a été considéré dans cette méthode. Une autre méthode intéressante appelé "méthode des tentacules" a été développée dans [Chebly(2015)]. L'idée est de déployer un ensemble de tentacules virtuelles avec différentes courbures devant le véhicule autonome, chaque tentacule représentant un chemin possible pour le véhicule. Chaque chemin est ensuite évalué par rapport à un ensemble de critères. L'avantage principal est que le véhicule est capable de naviguer sans "a priori" de l'environnement. Cependant, cette méthode peut conduire à des comportements difficiles à lire pour les usagers de la route.

Les méthodes des champs de potentiel sont souvent employées en robotique mobile ([Inarn(2012)], [Zhang(2017)], [Hu(2017)]...). Ces méthodes ont été également utilisées pour les "Unmanned Aircraft System" (UAV) dans [Zhan(2014)], [Andrew-Fields(2014)] et [Liu(2016)]. De plus, elles ont également été utilisées dans l'automobile, en particulier pour l'évitement d'obstacles dans [Shibata(2014)] cependant aucune extension pour une navigation complète n'a été développée.

Afin d'utiliser les champs de potentiel pour la navigation du véhicule autonome un schéma de contrôle a été développé (voir Figure 1). Ce schéma représente une architecture à trois étages :

- l'étage stratégique : les données sont collectées et traitées à partir des capteurs de perception afin de créer une carte virtuelle autour du véhicule autonome avec les informations sur les obstacles (vitesses, positions, ....) et les cibles ;

- l'étage tactique : à partir des informations précédentes, une force résultante de la combinaison des champs de potentiel répulsif et attractif est générée, puis, par l'intermédiaire d'un modèle simple (modèle masse ponctuelle), la position idéale, la vitesse et l'accélération sont générées en simulant le mouvement d'une particule chargée soumise à cette force générée ;

- l'étage opérationel : la position idéale, la vitesse et l'accélération venant de l'étage précédent sont utilisées comme références pour une boucle de contrôle classique, le contrôleur utilisant la différence entre références et mesures afin de génerer un angle volant $\beta_{w}$ et un angle roue $T_{w}$.

Dans ce papier, seulement l'étage tactique est présenté. En effet, l'étage stratégique est un problème de traitement de données tandis que l'étage opérationnel est un problème de contrôle. La dérivée non entière a déjà été utilisée dans l'automobile ([Morand(2016)]). Les travaux présentés dans ce papier sont basés sur une extension des travaux développés dans [Melchior(2001)], [Melchior(2003)],[Metoui(2009)] et [Moreau(2017)] sur les champs de potentiel fractionnaire. Dans un premier temps, la méthode des 
champs de potentiel fractionnaire est présentée en section 1. Ensuite, la section 2 montre les extensions de la méthode des champs de potentiel fractionnaire au véhicule autonome. Un ensemble de résultats de simulation est présenté en section 3, pour finalement conclure en section 4.

\section{Champs de potentiel fractionnaire}

En planification de trajectoire, une particule chargée est soumise à :

- un champ de potentiel attractif associé à la cible

- un champ de potentiel repulsif associé à chaque obstacle afin de l'éviter.

Comparée aux champs de potentiel classique, une définition fractionnaire du champ de potentiel permet de créer une courbure du champ de potentiel moins contrainte.

\subsection{Champs de potentiel répulsif fractionnaire}

Soit un champ de potentiel répulsif $U$ (voir Figure 2) définit par :

$$
U(r)=\frac{r^{n-2}-r_{\max }^{n-2}}{r_{\min }^{n-2}-r_{\max }^{n-2}} \quad \text { pour } \quad r_{\min }<r \leqslant r_{\max }
$$

où $r=\sqrt{\left(X_{o b s}-X\right)^{2}+\left(Y_{o b s}-Y\right)^{2}}$ est la distance entre la particule et l'obstacle considéré, $X_{o b s}$ et $Y_{o b s}$ sont les coordonnées de l'obstacle, enfin $r_{\min }$ et $r_{\max }$ sont les distances limites du champ potentiel répulsif et $n$ l'ordre fractionnaire. Le champ de potentiel peut être mis en forme par le biais du paramètre
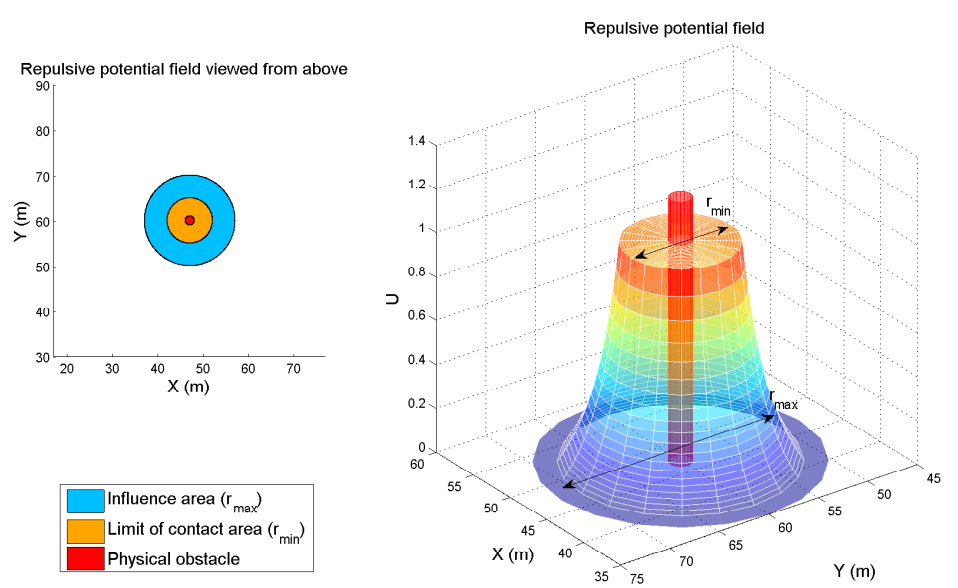

Figure 2. Champ de potentiel répulsif fractionnaire créé autour d'un obstacle positionné aux coordonnées $(45,60)$

$n$ (voir Fig. 3). Par exemple, quand un obstacle est sur la trajectoire d'une particule chargée, plus le paramètre $n$ est élevé, plus la particule va être repoussée par l'obstacle. Ainsi, le paramètre $n$ peut être interpreté comme un paramètre de danger. Le champ de potentiel est appliqué à chaque obstacle et le chemin libre est obtenu par troncature de la carte des potentiels ([Melchior(2003)]). La force résultante appliquée sur la particule est donnée par :

$$
\vec{F}=-\operatorname{grad}(\vec{U})
$$




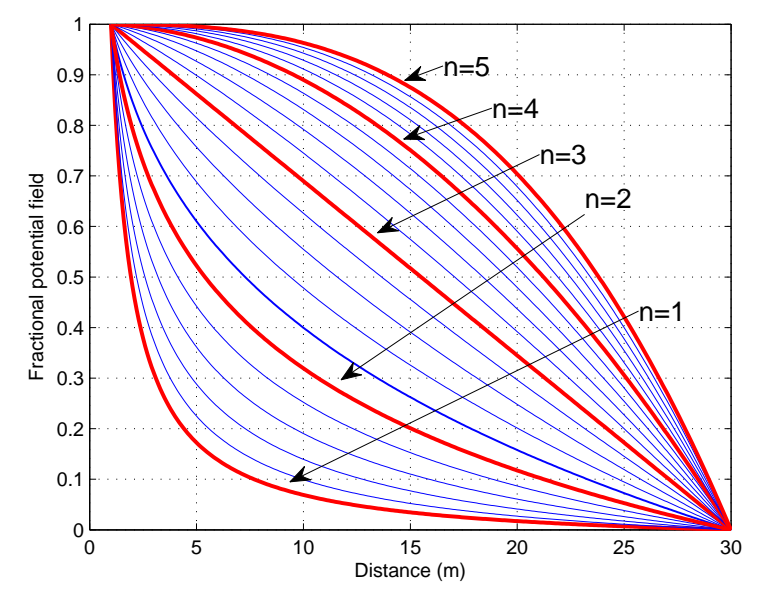

Figure 3. Influence du paramêtre $\mathrm{n}$ sur le champs de potentiel

\subsection{Champs de potentiel attractif fractionnaire}

La force attractive résultante exprimée par [Ge and Cui(2002)] est donnée par :

$$
\vec{F}=\alpha_{p}\left(\vec{x}_{\text {target }}-\vec{x}\right)+\alpha_{v}\left(\vec{v}_{\text {target }}-\vec{v}\right)
$$

où $\alpha_{p}$ et $\alpha_{v}$ sont des coefficients de pondération, la position de la particule $\vec{x}$, la positon de la cible $\vec{x}_{\text {target }}$, la vitesse de la particule $\vec{v}$ et la vitesse de la cible $\vec{v}_{\text {target }}$. Pour le champ de potentiel attractif fractionnaire, une extension de l'équation [1] donne :

$$
\vec{F}=\alpha_{p}\left(\vec{x}_{\text {target }}-\vec{x}\right)+\alpha_{v} \frac{d^{n}}{d t^{n}}\left(\vec{x}_{\text {target }}-\vec{x}\right),
$$

où la dérivée non entière de la position est considérée à la place de la vitesse.

Un modèle de masse ponctuelle peut être considéré de la forme :

$$
G(s)=\frac{X(s)}{F(s)}=\frac{1}{M s^{2}}
$$

où $s$ est la variable de Laplace, $X(s)$ est la transformée de Laplace de la position du véhicule et $F(s)$ la transformée de Laplace de la force résultante (répulsive et attractive).

Compte-tenu des équations [2] et [3], la force $F$ peut être considérée comme la commande du modèle masse ponctuelle, par conséquent l'équation [2] peut être reformulée comme un régulateur de position. Cette interprétation conduit au schéma de la boucle élémentaire de commande (voir Figure 4).

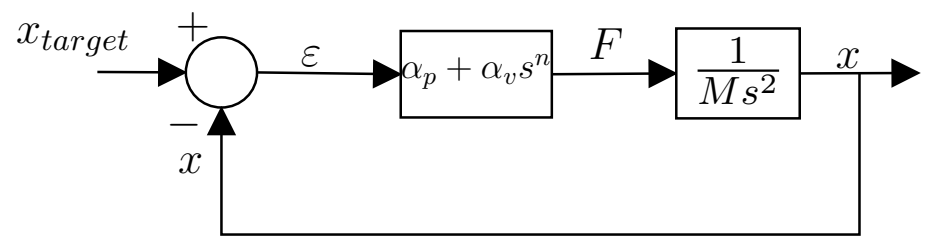

Figure 4. Schéma de boucle de contrôle élémentaire pour un champ de potentiel attractif de Ge\&Cui lorsque $n=1$ ou pour un champ de potentiel attractif fractionnaire dans les autres cas 


\subsection{Interprétation sur la boucle ouverte}

L'expression de la boucle ouverte associée à ce schéma s'écrit $\beta(j \omega)=\frac{\alpha_{v}(j \omega)^{n}+\alpha_{p}}{M(j \omega)^{2}}=\frac{1+\left(\frac{j \omega}{\omega_{c}}\right)^{n}}{\frac{M}{\alpha_{p}}(j \omega)^{2}}$. En hautes fréquences, elle devient :

$$
\beta(j \omega) \approx \frac{\alpha_{v}}{M(j \omega)^{2-n}}, \text { for } \omega \gg \omega_{c}=\left(\frac{\alpha_{p}}{\alpha_{v}}\right)^{\frac{1}{n}}
$$

ou encore,

$$
\beta(j \omega) \approx\left(\frac{\omega_{u g}}{j \omega}\right)^{n^{\prime}}
$$

où $n^{\prime}=2-n$ et la pulsation au gain unité :

$$
\omega_{u g}=\left(\frac{\alpha_{v}}{M}\right)^{\frac{1}{n^{\prime}}}
$$

Une telle boucle ouverte s'exprime par un ordre non entier $n^{\prime}$ et un coefficient d'amortissement donné par [Oustaloup(2014)] :

$$
\xi=-\cos \left(\frac{\pi}{n}\right)
$$

Dans ce cas, le coefficient d'amortissement ne dépend que du paramètre $n^{\prime}$ et est indépendant des paramètres du modèle. Ainsi, la robustesse du degré de stabilité est assurée malgré des variations de la masse du véhicule. Cette propriété de robustesse est inhérente à la forme de boucle ouverte et donc à la forme de la force attractive qui est de type proportionnel dérivé non entière.

Remarque 1 : La boucle ouverte possédant déjà deux actions intégrales, il n'est pas nécessaire d'ajouter une autre action intégrale dans le régulateur afin d'obtenir une erreur statique nulle et un rejet des perturbations de sortie.

Remarque 2: Si l'on s'intéresse au confort passager, il est nécessaire de minimiser l'accélération et le jerk. Cependant, comme expliqué précédemment, le fait d'ajouter un terme proportionnel à l'accélération et/ou au jerk entraine une modification de la forme de la boucle ouverte et ainsi peut entrainer une perte des propriétés de robustesse. Le jerk et l'accélération peuvent cependant être pris en compte dans l'ordre de dérivation non entière $n$. En effet, une dérivée de la position d'ordre $n$ compris entre 1 et 2 correspond à la prise en compte d'une combinaison de la vitesse et de l'accélération. Par extension, une dérivée de la position d'ordre $n$ compris entre 2 et 3 correspondrait à la prise en compte d'une combinaison de la l'accélération et du jerk [Oustaloup(2014)]. Néanmoins, les propriétés de stabilité de la boucle ouverte sont à étudier plus en détail. Le lecteur intéressé peut se référer à [Malti(2011)] pour connaître les propriétés de résonance et de stabilité des systèmes non entiers de second ordre généralisé. 


\section{Application au véhicule autonome}

Dans cette partie, les défauts du champ de potentiel attractif classique lorsqu'il est appliqué au véhicule autonome sont présentés. Une solution utilisant le concept de point de passage est proposée. Finalement, une amélioration de cette solution utilisant les champs de potentiel attractif fractionnaire est expliquée.

\subsection{Limites du champs de potentiel attractif de Ge\&Cui dans le contexte du véhicule auto- nome}

D’après la Figure 4, la transformée de Laplace de l'accélération peut être exprimée (avec $n=1$ ) conformément à :

$$
\Gamma(s)=\frac{\frac{\alpha_{v}}{\alpha_{p}} s^{3}+s^{2}}{\frac{M}{\alpha_{p}} s^{2}+\frac{\alpha_{v}}{\alpha_{p}} s+1} X_{\text {target }}(s) .
$$

Ainsi, deux problèmes principaux apparaissent avec une telle définition. Premièrement, cette fonction de transfert n'est pas propre (degré du numérateur supérieur à celui du dénominateur) ce qui conduit à des problèmes d'implantation. Le deuxième problème concerne la position de la cible : plus la cible est loin, plus l'amplitude de la force attractive est grande et donc plus l'accélération du véhicule est importante. Ainsi, si l'accélération du véhicule est trop importante, cela peut conduire à un inconfort pour les passagers et dans le pire cas, les limites du système peuvent être atteintes.

\subsection{Champs de potentiel attractif classique avec limitation de l'accélération du véhicule}

Tout d'abord, le problème de causalité peut être résolu en utilisant la vitesse du véhicule. En effet, la vitesse du véhicule est utilisée à la place de la dérivée de la position du véhicule. Cependant, la vitesse de la cible doit être ajoutée comme signal d'entrée comme illustrée sur la Figure 5.

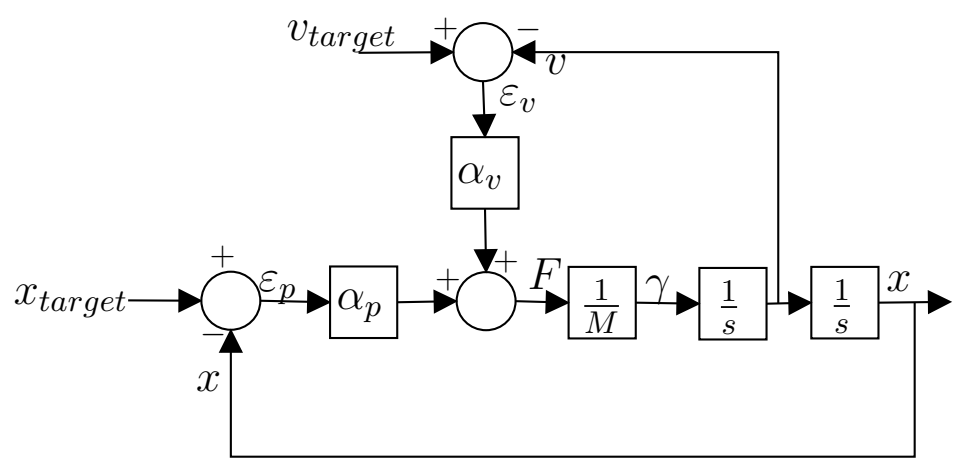

Figure 5. Schèma de la boucle de contrôle utilisant le champ de potentiel attractif et la vitesse

Ainsi, l'accélération du véhicule devient :

$$
\Gamma(s)=\frac{\frac{\alpha_{v}}{\alpha_{p}} s^{2}}{\frac{M}{\alpha_{p}} s^{2}+\frac{\alpha_{v}}{\alpha_{p}} s+1} V_{\text {target }}(s)+\frac{s^{2}}{\frac{M}{\alpha_{p}} s^{2}+\frac{\alpha_{v}}{\alpha_{p}} s+1} X_{\text {target }}(s) .
$$


Afin de limiter l'accélération à une certaine valeur $\gamma_{\max }$, l'équation (9) devient avec $s=j \omega$ :

$$
\begin{aligned}
\max _{\omega}|\Gamma(j \omega)| \leq \max _{\omega}\left|\frac{\frac{\alpha_{v}}{\alpha_{p}}(j \omega)^{2}}{\frac{M}{\alpha_{p}}(j \omega)^{2}+\frac{\alpha_{v}}{\alpha_{p}}(j \omega)+1}\right| & \left|V_{\text {target }}(j \omega)\right| \\
& +\max _{\omega}\left|\frac{(j \omega)^{2}}{\frac{M}{\alpha_{p}}(j \omega)^{2}+\frac{\alpha_{v}}{\alpha_{p}}(j \omega)+1}\right|\left|X_{\text {target }}(j \omega)\right|
\end{aligned}
$$

avec une cible stationnaire, $V_{\text {target }}=0$ et ainsi, les coefficients du champ de potentiel attractif peuvent être exprimés comme suit :

$$
\alpha_{v}=2 \xi \sqrt{\alpha_{p} M} \text { et } \alpha_{p} \leqslant \frac{\gamma_{\max } M}{\left|X_{\text {target }}\right|}
$$

Ces équations montrent que la cible doit être stationnaire et pas trop loin du véhicule afin de limiter son accélération. Conformément à ces contraintes, un ensemble de cibles peut être ajouté tout le long de l'itinéraire du véhicule. Ces cibles sont appelées points de passage. Le véhicule ne doit considérer que le point de passage le plus proche comme point attractif. C'est seulement après l'avoir atteint que le point suivant est considéré attractif. Il en va ainsi jusqu'à ce que le véhicule atteigne sa destination.

\subsection{Champs de potentiel attractif fractionnaire avec limitation de l'accélération du véhicule}

De la même manière que précedemment, un schéma similaire peut être établi dans le cas fractionnaire (voir Figure 6).

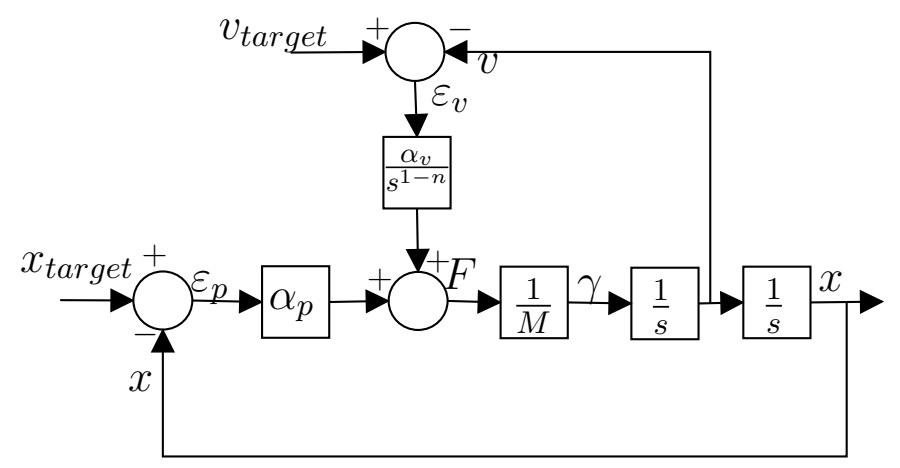

Figure 6. Champs de potentiel attractif fractionnaire avec causalité

Dans ce cas, l'accélération du véhicule devient :

$$
\Gamma(s)=\frac{\frac{\alpha_{v}}{\alpha_{p}} s^{n+1}}{\frac{M}{\alpha_{p}} s^{2}+\frac{\alpha_{v}}{\alpha_{p}} s^{n}+1} V_{\text {target }}(s)+\frac{s^{2}}{\frac{M}{\alpha_{p}} s^{2}+\frac{\alpha_{v}}{\alpha_{p}} s^{n}+1} X_{\text {target }}(s) .
$$

Afin de limiter l'accélération à une valeur $\gamma_{\max }$, l'équation (12) devient alors : 


$$
\begin{aligned}
\max _{\omega}|\Gamma(j \omega)| \leqslant \max _{\omega}\left|\frac{\frac{\alpha_{v}}{\alpha_{p}}(j \omega)^{n+1}}{\frac{M}{\alpha_{p}}(j \omega)^{2}+\frac{\alpha_{v}}{\alpha_{p}}(j \omega)^{n}+1}\right|\left|V_{\text {target }}(j \omega)\right|+ \\
\max _{\omega}\left|\frac{(j \omega)^{2}}{\frac{M}{\alpha_{p}}(j \omega)^{2}+\frac{\alpha_{v}}{\alpha_{p}}(j \omega)^{n}+1}\right|\left|X_{\text {target }}(j \omega)\right| .
\end{aligned}
$$

En considérant une cible stationnaire, $V_{\text {target }}=0$ et l'équation (6), l'expression des coefficients du champ de potentiel attractif s'expriment :

$$
\alpha_{v}=M \omega_{u g}^{n^{\prime}} \text { et } \alpha_{p} \leqslant \frac{\gamma_{\max } M}{\left|X_{\text {target }}\right|} .
$$

Il est possible d'aller plus loin dans des considérations de confort en s'intéressant au jerk. Cependant, de telles considérations conduiraient à une modification de la forme de la force attractive et donc à une perte de la propriété de robustesse du degré de stabilité.

\subsection{Potentiel attractif en vitesse de Ge\&Cui}

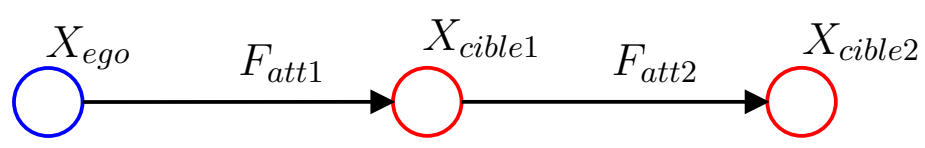

Figure 7. Schéma de principe d'utilisation des points de passage

Les paragraphes précédents ont permis de montrer les améliorations apportées à la théorie des champs de potentiels de Ge\&Cui. Cependant, suite à l'introduction des points des passages, il est nécessaire de considérer un ensemble de cibles attractives. Nous considérons que l'attractivité d'une cible de position $k+1$ est ressentie par l'égo-véhicule lorsque la cible de position $k$ a été atteinte. Dans ce paragraphe, le scénario simple à deux points de passage est considéré conformément à la Figure 7.

Une simulation avec changement de position de cible est alors effectuée. La cible $\mathrm{n}^{\circ} 2$ est considérée seulement lorsque la cible $\mathrm{n}^{\circ} 1$ a été atteinte par l'égo-véhicule. La première cible est positionnée à $100 \mathrm{~m}$ de l'égo véhicule tandis que la deuxième est positionnée à $200 \mathrm{~m}$. Les résultats de cette simulation sont présentés en Figure 8. On constate que le véhicule atteint bien sa destination tout en ayant une
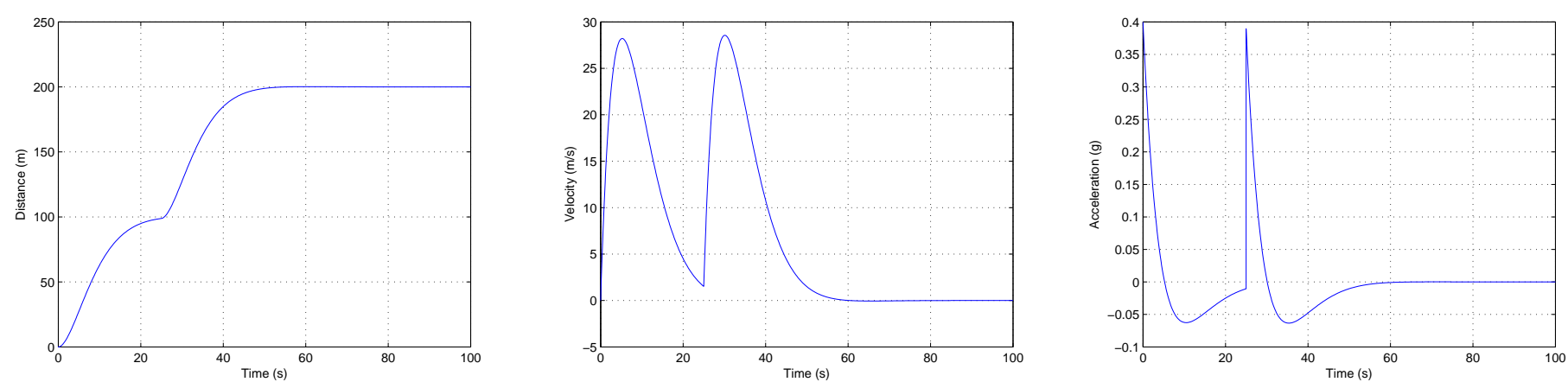

Figure 8. Évolution de la position (gauche), de la vitesse (milieu) et de l'accélération (droite) du véhicule autonome lors du passage consécutif de deux points de passage 
accélération inférieure à la contrainte fixée à $0.5 \mathrm{~g}$, où g est l'accélération de la gravité. Cependant, on peut observer un profil d'accélération en dent de scie ainsi qu'un profil de vitesse s'annulant au point de passage intermédiaire. Ces observations peuvent être considérées comme des effets indésirables pour les passagers. Il est donc nécessaire, dans un premier temps, de comprendre ces phénomènes afin de les corriger dans un deuxième temps.

En intégrant la relation (9), on obtient l'expression de la vitesse de l'égo-véhicule en fonction de la vitesse de la cible :

$$
V(s)=\frac{\frac{\alpha_{v}}{\alpha_{p}} s}{\frac{M}{\alpha_{p}} s^{2}+\frac{\alpha_{v}}{\alpha_{p}} s+1} V_{\text {target }}(s)+\frac{s}{\frac{M}{\alpha_{p}} s^{2}+\frac{\alpha_{v}}{\alpha_{p}} s+1} X_{\text {target }}(s) .
$$

En utilisant les théorèmes de la valeur initiale et de la valeur finale, on constate que la vitesse du véhicule s'annule dans les premiers et derniers instants et ce, quelles que soit la vitesse et la position de la cible considérée. Cette constatation est assez logique dans le sens où le principe du potentiel attractif est de minimiser l'erreur de position entre la cible et le véhicule. Cependant, il n'est pas possible de minimiser cette erreur en considérant une vitesse du véhicule non nulle ainsi qu'une cible fixe.

En prenant en considération les contraintes du code de la route notamment en termes de limitation de vitesse, il serait intéressant de pouvoir imposer une certaine vitesse. Pour faire cela, on considère la dérivée de la force attractive de Ge\&Cui conformément à l'équation suivante :

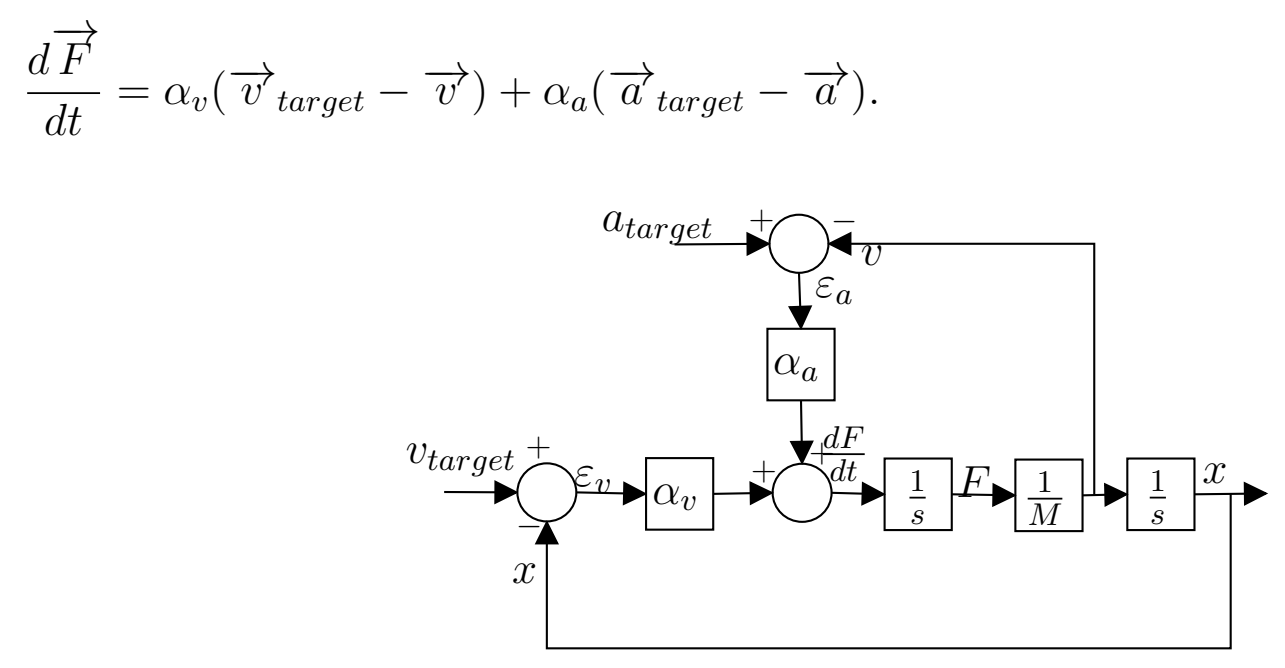

Figure 9. Schèma de la boucle de contrôle utilisant le champ de potentiel attractif et la vitesse

Cette équation conduit à l'architecture de la Figure 9. On constate que cette architecture est similaire à la Figure 5 présentée au paragraphe 2.2. Seuls les signaux sont différents (dérivées des signaux considérés dans l'architecture précédente) et un intégrateur a été échangé avec le coefficient $1 / M$.

On peut alors écrire les équations relatives à la vitesse du véhicule :

$$
V(s)=\frac{\frac{\alpha_{a}}{\alpha_{v}}}{\frac{M}{\alpha_{v}} s^{2}+\frac{\alpha_{a}}{\alpha_{v}} s+1} A_{\text {target }}(s)+\frac{s}{\frac{M}{\alpha_{v}} s^{2}+\frac{\alpha_{a}}{\alpha_{v}} s+1} V_{\text {target }}(s)
$$



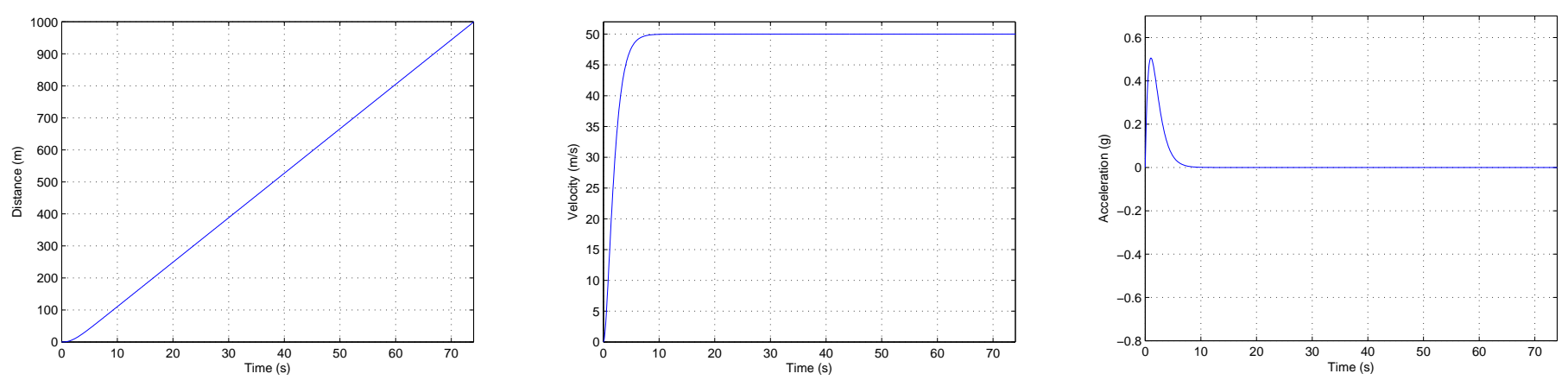

Figure 10. Évolution de la position (gauche), de la vitesse (milieu) et de l'accélération (droite) du véhicule autonome lors du passage consécutif de deux points de passage en utilisant le potentiel attractif en vitesse

et à l'accélération du véhicule :

$$
\Gamma(s)=\frac{\frac{\alpha_{v}}{\alpha_{p}} s}{\frac{M}{\alpha_{p}} s^{2}+\frac{\alpha_{v}}{\alpha_{p}} s+1} V_{\text {target }}(s)+\frac{s}{\frac{M}{\alpha_{p}} s^{2}+\frac{\alpha_{v}}{\alpha_{p}} s+1} X_{\text {target }}(s) .
$$

Ces équations permettent d'obtenir les nouvelles expressions des coefficients de Ge\&Cui :

$$
\alpha_{a}=2 \xi \sqrt{\alpha_{v} M} \text { et } \alpha_{v} \leq \frac{2 \xi M V_{\max }}{\gamma_{\max }}
$$

Une simulation en ligne droite est ensuite effectuée. Le point de passage attractif est placé au point de coordonnées $(1000 \mathrm{~m}, 1000 \mathrm{~m})$ alors que le véhicule est placé à l'origine du repère $(0 \mathrm{~m}, 0 \mathrm{~m})$.

Les Figures 10 représentent respectivement la position, la vitesse et l'accélération du véhicule selon l'axe X. La simulation étant en ligne droite et le point de passage étant équidistant de l'origine que çe soit en $\mathrm{X}$ ou en $\mathrm{Y}$, seules les réponses temporelles selon l'axe $\mathrm{X}$ sont présentées. On constate que le véhicule atteint sa destination en $75 \mathrm{~s}$ avec une vitesse de $50 \mathrm{~km} / \mathrm{h}$ ainsi qu'avec une accélération maximum de $0.5 \mathrm{~g}$ conformément aux contraintes d'accélération fixée. On peut donc en conclure qu'en utilisant un tel potentiel, nous sommes capable d'imposer une vitesse tout en limitant l'accélération. Cependant, la position n'étant plus minimisée et la vitesse étant non nulle, le véhicule ne s'arrête pas, il faut donc complexifier cette méthode.

\subsection{Potentiel attractif de Ge\&Cui mixte}

Dans le paragraphe précédent, nous avons introduit un champ de potentiel permettant d'imposer une vitesse. Cette méthode est tout à fait adaptée pour une navigation du véhicule sur un tronçon de route limité par une certaine vitesse. Cependant elle ne permet pas de faire converger le véhicule vers une position donnée contrairement à la méthode classique de champ de potentiel attractif. Au vu de cette constatation, il semble judicieux de combiner ces deux méthodes permettant ainsi de palliers leurs défauts respectifs et ainsi de profiter de leurs avantages. Pour ce faire, il est nécessaire de définir deux types de points de passages : le premier est appelé point de passage de transition imposant une vitesse au véhicule tandis que le deuxième est appelé point de passage d'arrêt imposant une position au véhicule. Ainsi lorsque le prochain point de passage considéré par le véhicule est un point de passage de transition, le véhicule reçoit une référence en vitesse et fait appel au champ de potentiel attractif en vitesse. De même, 
lorsque le prochain point de passage considéré par le véhicule est un point de passage d'arrêt, le véhicule reçoit une référence en position et fait appel au champ de potentiel attractif classique.
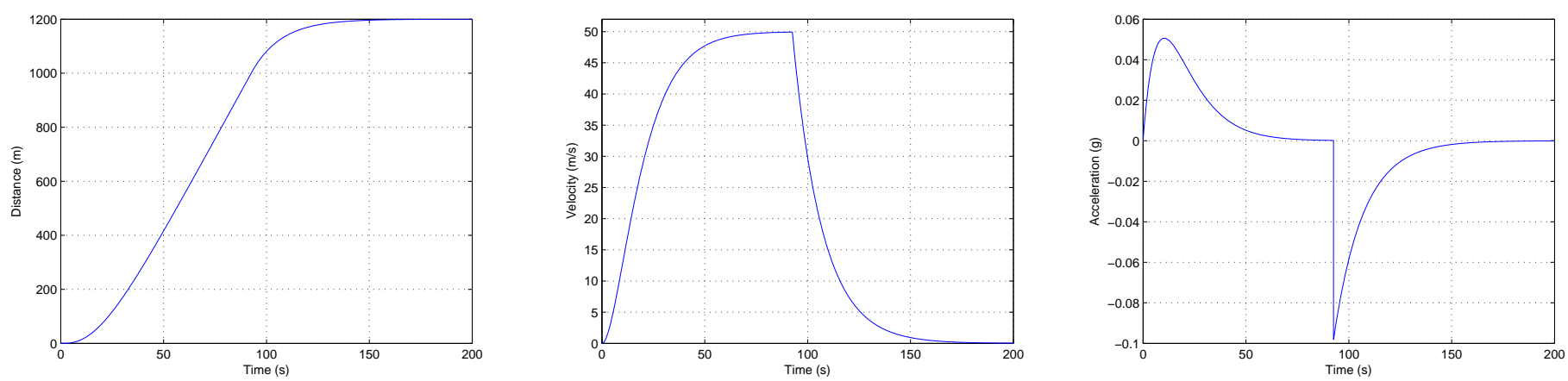

Figure 11. Évolution de la position (gauche), de la vitesse (milieu) et de l'accélération (droite) du véhicule autonome lors du passage consécutif de deux points de passage en utilisant le potentiel attractif mixte

On considère le scénario suivant : le véhicule circule en ligne droite. Un point de passage de transition est placé à $1000 \mathrm{~m}$ du véhicule et un point de passage d'arrêt est placé encore $200 \mathrm{~m}$ plus loin. Les résultats de simulation sont présentés en Figure 11. On constate que le véhicule parcours le premier tronçon à une vitesse de $50 \mathrm{~km} / \mathrm{h}$ avec une accélération inférieure à $0.5 \mathrm{~g}$ et s'arrête au point de passage d'arrêt conformément à nos attentes.

\section{Accélération contrainte et étude de robustesse}

\subsection{Simulation de l'accélération contrainte}

Le véhicule autonome est attiré par un point de passage unique conformément à la théorie des champs de potentiel attractif. Les coefficients du champ de potentiel attractif sont choisis en accord avec l'expression [11] où $\xi=1$ (sans dépassement), $M=750 \mathrm{~kg}$ (véhicule léger) et le point de passage est placé $100 \mathrm{~m}$ plus loin que le véhicule. Une limite de l'accélération est fixée à $0.5 \mathrm{~g}$. Le diagramme de Bode de la fonction de sensibilité $\frac{\Gamma(s)}{X_{\text {target }}(s)}$ (courbe bleue) et la contrainte d'accélération (courbe verte) sont tracées en Figure 12. Comme attendu, ce choix de coefficient respecte la contrainte d'accélération dans la bande de fréquences utile.

Afin d'être certain que cette contrainte est bel et bien respectée, une simulation en ligne droite est effectuée. Le point attractif est placé aux coordonnées $(100 \mathrm{~m}, 100 \mathrm{~m})$ tandis que le véhicule est placé à l'origine du repère $(0 \mathrm{~m}, 0 \mathrm{~m})$. Les figures 13 , représentent respectivement la position, la vitesse et l'accélération du véhicule relatives à l'axe X. La simulation étant en ligne droite et le point attractif étant à la même distance en $\mathrm{X}$ ou en $\mathrm{Y}$ de l'origine, seules les réponses temporelles relatives à la coordonnée $\mathrm{X}$ sont présentées. Il est à noter que le véhicule atteint sa destination en $30 \mathrm{~s}$ avec une vitesse maximum de $30 \mathrm{~km} / \mathrm{h}$ et une accélération maximum de $0.5 \mathrm{~g}$ conformément à la contrainte d'accélération. Si un obstacle apparaît durant le mouvement, la trajectoire sera localement ajustée afin de l'éviter. 


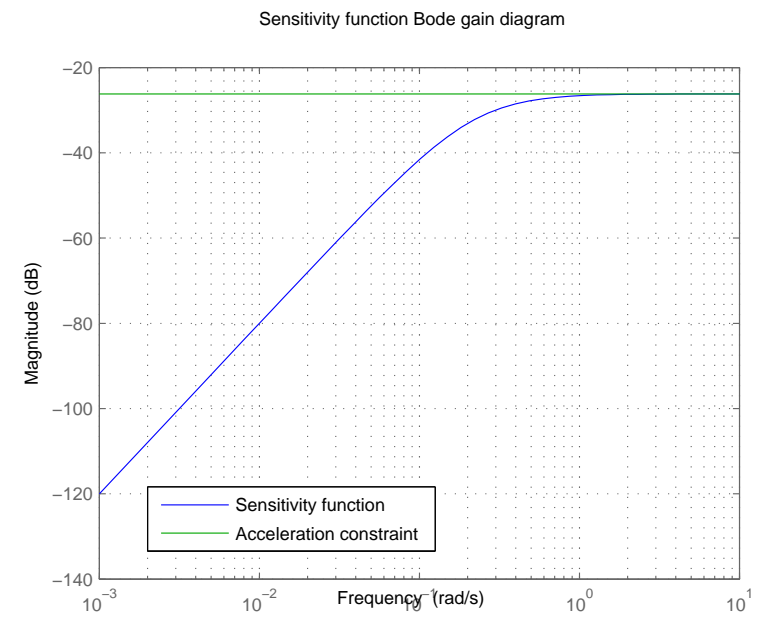

Figure 12. Gain de la fonction de sensibilité
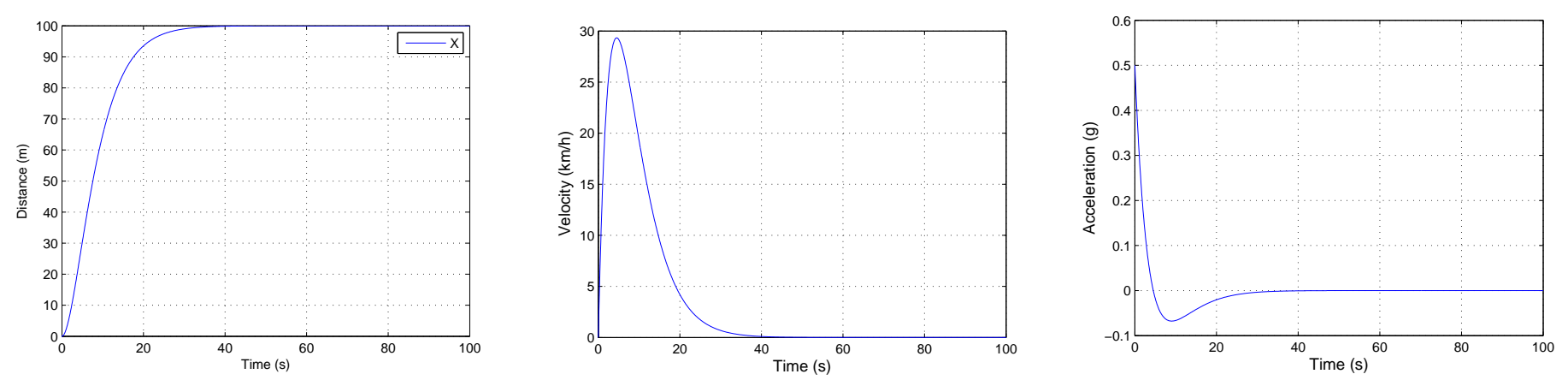

Figure 13. Évolution de la position (gauche), de la vitesse (milieu) et de l'accélération (droite) du véhicule

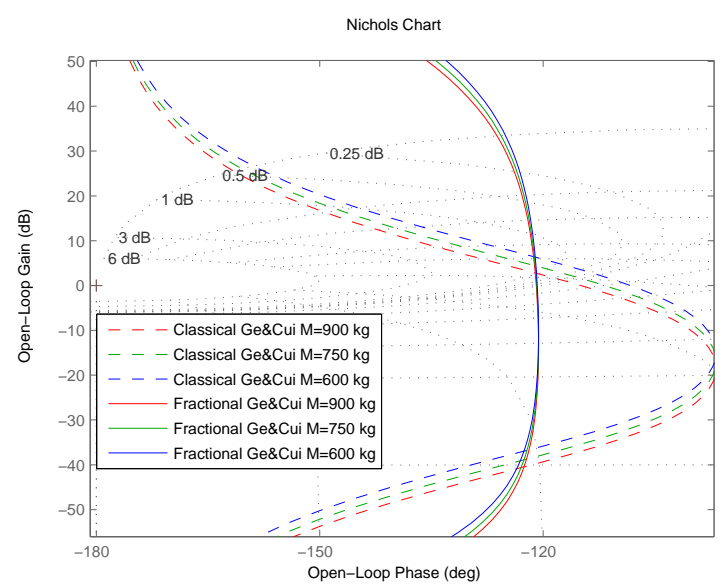

Figure 14. Diagramme de Nichols de la boucle ouverte fractionnaire et de la boucle ouverte classique

\subsection{Simulation de la robustesse du degré de stabilité}

Maintenant, la masse du véhicule varie (un conducteur, plusieurs passagers, un véhicule complétement chargé,...). Les champs de potentiel attractif classique et fractionnaire sont paramétrés pour une masse maximum et avec une même pulsation au gain unité $\omega_{u g}=0.39 \mathrm{rad} / \mathrm{s}$ conformément à la relation [4]. Ensuite, l'impact des variations de masse sur le degré de stabilité est testé. La Figure. 14 représente le 

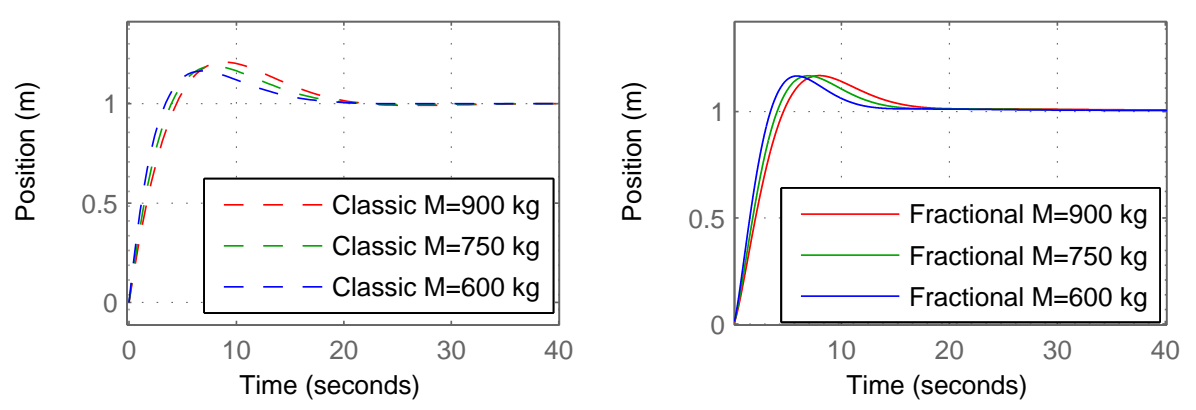

Step Response (force)
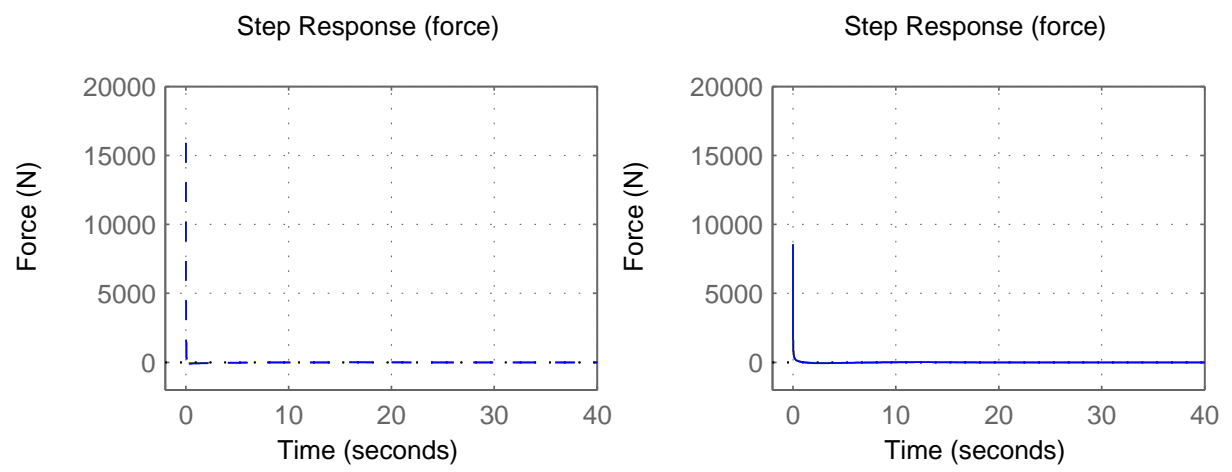

Figure 15. Illustration de la robustesse dans le cas fractionnaire (droite) comparée au cas classique (gauche)

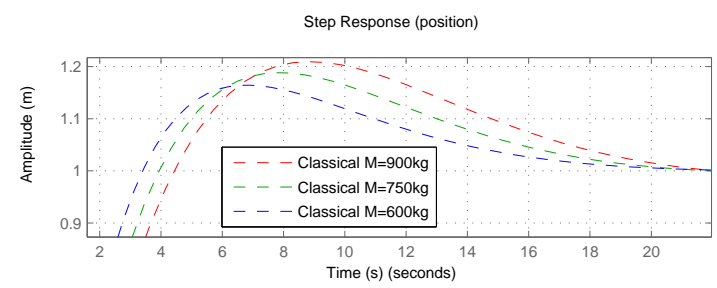

Step Response (position)

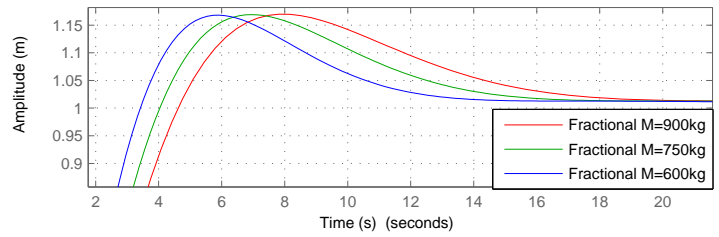

Figure 16. Illustration de la robustesse dans le cas fractionnaire (bas) comparée au cas classique (haut)

diagramme de Nichols des boucles ouvertes pour les cas classiques (pointillés) et fractionnaires (traits pleins) pour une masse maximum $M=900 \mathrm{~kg}$ (rouge), une masse moyenne $M=750 \mathrm{~kg}$ (vert) et une masse minimum $M=600 \mathrm{~kg}$ (bleu). Dans le cas classique, les variations de masse conduisent à des variations de phase autour de la pulsation au gain unité $\omega_{u g}$ (la marge de phase varie entre $65^{\circ}$ et $70^{\circ}$ ) contrairement au cas fractionnaire où il n'y a pas de variations autour de $\omega_{u g}$ (la marge de phase est verrouillée à $60^{\circ}$ ). Ainsi, dans le cas fractionnaire, la marge de phase est maintenue, assurant ainsi la robustesse du degré de stabilité.

Les Figures 15 et les Figures 16 présentent respectivement les réponses indicielles de la position pour le cas classique (pointillés) et dans le cas fractionnaire (trait plein) pour une masse maximum (rouge), 


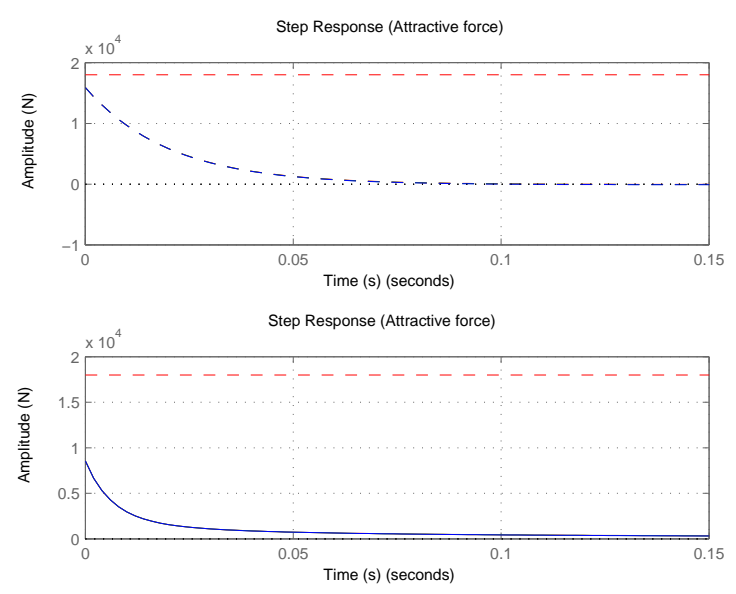

Figure 17. Illustration de la robustesse dans le cas fractionnaire (bas) comparée au cas classique (haut) avec la force attractive correspondant (bleu) et la contrainte de force (rouge)

une masse moyenne (vert) et une masse minimum (bleu). Dans le cas fractionnaire, le dépassement est maintenu constant lorsque la masse varie contrairement au cas classique. Finalement, la Figure 17 illustre la force attractive correspondante (bleu) avec la contrainte de force (rouge). Dans les deux cas, la contrainte de force est respectée cependant, dans le cas fractionnaire, la courbe est bien en dessous de cette limite comparée au cas classique. De plus, le temps de réponse du champ de potentiel attractif fractionnaire peut être amélioré contrairement au cas classique.

\section{Conclusion et perspectives}

Les travaux de recherche sur le véhicule autonome conduisent les fabricants de véhicule à se concentrer sur les zones urbaines. Cet environnement est un point critique pour le véhicule autonome car il est très variable du fait de la diversité des infrastructures routières (intersections, carrefour giratoire, ...). Ainsi, il est essentiel de développer une planification de trajectoires adaptative par rapport à l'environnement du véhicule autonome.

De plus, un état de l'art non exhaustif de ces méthodes permet de positionner la méthode des champs de potentiel par rapport à l'existant. Le principal avantage de cette méthode est son adaptibilité ce qui est un vrai besoin pour le véhicule autonome. Cependant, cette méthode requiert quelques modifications afin de devenir complètement applicable au véhicule autonome.

À partir de cette perspective, une adaptation du potentiel attractive est proposée permettant de limiter l'accélération, d'imposer la vitesse et de garantir la robustesse du degré de stabilité. Un ensemble de points attractifs appelés points de passage sont aussi introduits afin de mieux répondre aux contraintes du véhicule autonome.

L'adaptation de la méthode des champs de potentiel attractif est résolue dans ce papier, le répulsif, quant à lui doit être mis à l'échelle vis-a-vis de l'attractif. De plus, des scénarios de situations de vie doivent être simulés et validés avant de tester cette stratégie sur un véritable véhicule. 


\section{Bibliographie}

A. Chebly, G. Tagne, R.T. and Charara, A. (2015). Local trajectory planning and tracking for autonomous vehicle navigation using clothoid tentacles method. In proceedings of International IEEE Conference on Intelligent Vehicles Symposium (IV), Seoul, Korea.

Andrew-Fields, R. (2014). Continuous Control Artificial Potential Function Methods and Optimal Control. Ph.D. thesis, Air Force Institute of Technology.

Ge, S. and Cui, Y. (2002). Dynamic motion planning for mobile robots using potential field method. Autonomous Robots, $13,207-222$.

Hu, X., Li, Z., and CAO, J. (2017). A Path Planning Method Based on Artificial Potential Field Improved by Potential Flow Theory. 2nd International Conference on Computer Science and Technology (CST 2017), Vienna, Austria.

Inarn, C., Melchior, P., Metoui, B., and Oustaloup, A. (2012). Robust path planning for 3D dynamic environment based on fractional attractive force. 5th IFAC Symposium on Fractional Differentiation and Its Applications (IFAC FDA'12), Grenoble, France.

Lefèvre, S. (2013). Risk Estimation at Road Intersections for Connected Vehicle Safety Applications. Ph.D. thesis, Université de Grenoble.

Liu, Y., Zhang, X., Guan, X., and Delahayel, D. (2016). Potential odor intensity grid based UAV path planning algorithm with particle swarm optimization approach. Mathematical Problems in Engineering, 2016, 1-16.

Makarem, L. and Gillet, D. (2011). Decentralized coordination of autonomous vehicles. In proceedings of the 18th IFAC World Congress, Milan, Italy, 44, 13046-13051.

Malti, R., Moreau, X., Khemane, F., and Oustaloup, A. (2011). Stability and resonance conditions of elementary fractional transfer functions. Automatica, 47, 2462-2467.

Melchior, P., Orsoni, B., Lavialle, O., Poty, A., and Oustaloup, A. (2003). Consideration of obstacle danger level in path planning using $A^{*}$ and fast-marching optimisation : comparative study. Signal processing, 83, 2387-2396.

Melchior, P., Orsoni, B., and Oustaloup, A. (2001). Weyl fractional potential in path planning. Proceedings of the Sixth IEEE ECC'2001, Porto, Portugual.

Metoui, B., Melchior, P., Najar, S., Abdelkrim, M., and Oustaloup, A. (2009). Robust path planning for dynamic environment based on fractional attractive force. Sixth IEEE International Multi-Conference on Systems, Signals and Devices (IEEE SSD'09), Djerba, Tunisia.

Morand, A., Moreau, X., Melchior, P., Moze, M., and Guillemard, F. (2016). Crone cruise control system. IEEE Transactions on Vehicular Technology, 65, 15-28.

Moreau, J., Melchior, P., Victor, S., Aioun, F., and Guillemard, F. (2017). Path planning with fractional potential fields for autonomous vehicles. IFAC-PapersOnLine, 50, 14533-14538.

Oustaloup, A. (2014). Diversity and Non-integer Differentiation for System Dynamics. Wiley-ISTE.

Shibata, N., Sugiyama, S., and Wada, T. (2014). Collision avoidance control with steering using velocity potential field. IEEE Intelligent Vehicles Symposium, Ypsilanti, Michigan, USA.

Zhan, W., Wang, W., Chen, N., and Wang, C. (2014). Efficient UAV path planning with multiconstraints in a 3D battlefield environnement. Mathematical Problems in Engineering, 2014, 1-12.

Zhang, Y., Liu, Z., and Chang, L. (2017). A New Adaptative Artificial Potential Field and Rolling Window Method for Mobile Robot Path Planning. 29th Chinese Control And Decision Conference (CCDC'17), Chongqing, China. 